\title{
The effect of Gamma Knife radiosurgery on large posterior fossa metastases and the associated mass effect from peritumoral edema
}

\author{
*Baha'eddin A. Muhsen, MD,, ${ }^{1,3}$ Krishna C. Joshi, MD, MCh, ${ }^{1,3}$ Bryan S. Lee, MD, ${ }^{1}$ Bicky Thapa, MD, ${ }^{3}$ \\ Hamid Borghei-Razavi, MD, ${ }^{1,3}$ Xuefei Jia, ${ }^{2}$ Gene H. Barnett, MD, ${ }^{1,3}$ Samuel T. Chao, MD, ${ }^{3,4}$ \\ Alireza M. Mohammadi, MD, , John H. Suh, MD, 3,4 Michael A. Vogelbaum, MD, PhD, ${ }^{1,3}$ and \\ Lilyana Angelov, MD1,3
}

\begin{abstract}
1Department of Neurosurgery, Cleveland Clinic Lerner College of Medicine of Case Western Reserve University, Cleveland Clinic, Cleveland; ${ }^{2}$ Quantitative Health Sciences, Taussig Cancer Institute, Cleveland Clinic, Cleveland; ${ }^{3}$ Rose Ella Burkhardt Brain Tumor and Neuro-Oncology Center, Neurological Institute, Cleveland Clinic, Cleveland; and ${ }^{4}$ Department of Radiation Oncology, Taussig Cancer Institute, Cleveland Clinic, Cleveland, Ohio
\end{abstract}

OBJECTIVE Gamma Knife radiosurgery (GKRS) as monotherapy is an option for the treatment of large $(\geq 2 \mathrm{~cm})$ posterior fossa brain metastases (LPFMs). However, there is concern regarding possible posttreatment increase in peritumoral edema (PTE) and associated compression of the fourth ventricle. This study evaluated the effects and safety of GKRS on tumor and PTE control in LPFM.

METHODS The authors performed a single-center retrospective review of 49 patients with 51 LPFMs treated with GKRS. Patients with at least 1 clinical and radiological follow-up visit were included. Tumor, PTE, and fourth ventricle volumetric measurements were used to assess efficacy and safety. Overall survival was a secondary outcome.

RESULTS Fifty-one lesions in 49 consecutive patients were identified; $57.1 \%$ of patients were male. At the time of GKRS, the median age was 61.5 years, and the median Karnofsky Performance Status score was 90 . The median number of LPFMs and overall brain metastases were 1 and 2, respectively. The median overall tumor, PTE, and fourth ventricle volumes at diagnosis were $4.96 \mathrm{~cm}^{3}$ (range 1.4-21.1 $\left.\mathrm{cm}^{3}\right), 14.98 \mathrm{~cm}^{3}$ (range $\left.0.6-71.8 \mathrm{~cm}^{3}\right)$, and $1.23 \mathrm{~cm}^{3}($ range $0.3-3.2 \mathrm{~cm}^{3}$ ), respectively, and the median lesion diameter was $2.6 \mathrm{~cm}$ (range $2.0-5.07 \mathrm{~cm}$ ). The median follow-up time was 7.3 months (range 1.6-57.2 months). At the first follow-up, 2 months posttreatment, the median tumor volume decreased by $58.66 \%$ (range $-96.95 \%$ to $+48.69 \%$, $p<0.001$ ), median PTE decreased by $78.10 \%$ (range $-99.92 \%$ to $+198.35 \%, p<0.001$ ), and the fourth ventricle increased by $24.97 \%$ (range $-37.96 \%$ to $+545.6 \%, p<0.001$ ). The local control rate at first follow-up was $98.1 \%$. The median OS was 8.36 months. No patient required surgical intervention, external ventricular drainage, or shunting between treatment and first follow-up. However, 1 patient required a ventriculoperitoneal shunt at 23 months from treatment. Posttreatment, $65.30 \%$ received our general steroid taper, $6.12 \%$ received no steroids, and $28.58 \%$ required prolonged steroid treatment.

CONCLUSIONS In this retrospective analysis, patients with LPFMs treated with GKRS had a statistically significant posttreatment reduction in tumor size and PTE and marked opening of the fourth ventricle (all $p<0.001)$. This study demonstrates that GKRS is well tolerated and can be considered in the management of select cases of LPFMs, especially in patients who are poor surgical candidates.

https://thejns.org/doi/abs/10.3171/2019.11.JNS191485

KEYWORDS peritumoral edema; large posterior fossa metastases; Gamma Knife radiosurgery; brain metastasis; whole-brain radiotherapy; oncology; stereotactic radiosurgery

\footnotetext{
ABBREVIATIONS GKRS = Gamma Knife radiosurgery; KPS = Karnofsky Performance Status; LPFM = large posterior fossa brain metastasis; OS = overall survival; PTE = peritumoral edema; RCC = renal cell carcinoma; SRS = stereotactic radiosurgery; STM = supratentorial metastasis; WBRT = whole-brain radiotherapy. SUBMITTED May 26, 2019. ACCEPTED November 12, 2019.

INCLUDE WHEN CITING Published online January 24, 2020; DOI: 10.3171/2019.11.JNS191485.

${ }^{*}$ B.A.M. and K.C.J. share first authorship of this work.
} 
$\mathrm{B}$ RAIN metastases are the most common cause of intracranial neoplasms and typically arise from the lung, breast, and skin. ${ }^{42}$ They occur in approximately $20 \%-40 \%$ of all patients with cancer and are associated with higher patient mortality and morbidity due to the extensive local and systemic therapy required. Various treatment options and modalities must be evaluated and carefully chosen in order to optimize the management of the intracranial disease and the patient's quality of life., ${ }^{9,14}$ Stereotactic radiosurgery (SRS), such as Gamma Knife radiosurgery (GKRS), is an effective treatment modality for brain metastases and has been reported to demonstrate efficacy in previous trials either as monotherapy or in conjunction with whole-brain radiotherapy (WBRT)., 2,3,20,33, ${ }_{41,43}$ Monotherapy with GKRS has recently gained favor as it is associated with fewer toxicities and neurocognitive side effects in comparison with WBRT alone, or combined GKRS therapy with WBRT. $8,11,22,28,39$

The effect of radiosurgery on control of peritumoral edema (PTE), however, is not well understood. ${ }^{25,37,38}$ It is known that PTE around brain metastases can lead to various neurological sequelae depending on the location and the severity of the edema. ${ }^{33,37}$ One significant concern is that large metastases located in the posterior fossa when associated with significant PTE have the potential to compress the brainstem and obliterate the CSF pathways, with resultant rapid neurological deterioration and a high risk of mortality. High-dose steroids can initially be used to prevent acute neurological deterioration secondary to the mass effect induced by significant PTE., ${ }^{7,32,37}$ However, there is a paucity of literature on the effect of GKRS on PTE, and neurosurgeons are inclined to favor resection of large ( $\geq 2$ $\mathrm{cm}$ ) posterior fossa brain metastases (LPFMs) with PTE to prevent devastating neurological sequelae secondary to the worsening of PTE and the associated mass effect. ${ }^{1,7,16,17}$

A limited number of studies in the literature have addressed the effect of GKRS on PTE around cerebral metastases. ${ }^{18,23,27,37,38}$ The lesions evaluated in these previous studies were heterogeneous with respect to the size, location, presence of tumor-associated hemorrhage, and treatment received (WBRT with or without surgery). None of these studies additionally analyzed the effect of GKRS on PTE around LPFMs. The aim of our study was to objectively evaluate the effect of GKRS on LPFMs and associated PTE as well as the associated mass effect on the adjacent fourth ventricle, thereby improving our understanding of the treatment outcomes of GKRS in this group of patients and enhancing our decision-making process in the management of large tumors in this challenging location.

\section{Methods}

This was an IRB-approved retrospective chart review evaluating patients with LPFMs (defined as $\geq 2 \mathrm{~cm}$ in the largest diameter, based on previously published series ${ }^{4}$ ) who underwent GKRS in a single fraction at our institution. We identified 51 lesions in 49 patients treated from January 1, 2009, to December 31, 2017. Patients were included in the study if they were 18 years or older, had at least 1 LPFM, and had at least 1 clinical and radiological follow-up after GKRS. Those who underwent surgi- cal excision of these lesions and those with evidence of intratumoral hemorrhage either at the time of diagnosis or at first follow-up were excluded from the study, as the hemorrhage could potentially alter the total volume of the tumor and PTE, and moreover it could change the MRI signal intensity around the lesions. Prior WBRT (20 patients, $40.8 \%$ ) was not an exclusion criterion. The presence or absence of PTE was neither an inclusion nor exclusion criterion. None of the patients received subsequent or staged GKRS to the lesion. Also, patients were excluded if they had any clinical or radiological evidence of leptomeningeal disease. The detailed inclusion and exclusion criteria for our study are summarized in Supplementary Table 1.

The following data were obtained from medical records: patient demographics, clinical presentation, Karnofsky Performance Status (KPS) score, lesion diameter (in $\mathrm{cm}$ ) and volume (in $\mathrm{cm}^{3}$ ), number of metastases in the posterior fossa, details of WBRT, GKRS prescribed dose, and duration of posttreatment follow-up. GKRS was performed using the Leksell Gamma Knife Perfexion (during the initial study period; Elekta $\mathrm{AB}$ ) and the Icon (post-March 2017). Pre-GKRS and post-GKRS volumetric measurements of the tumor and fourth ventricle were performed using gadolinium-enhanced T1-weighted MRI sequences, and PTE volume was measured on T2-weighted FLAIR sequences using Brainlab software (iPlan Net 3.6.0) (Fig. 1). When contouring the fourth ventricle on iPlan, the superior extent of the fourth ventricle was defined as the inferior-most border of the sylvian aqueduct, and the inferior extent of the fourth ventricle was the foramen of Magendie. On either side, the foramen of Luschka was considered as the lateral extent of the fourth ventricle. ${ }^{24}$ In each patient, the presence of any hydrocephalus was assessed by calculating the ratio of the maximal dimensions of the frontal horn to the distance between the inner diameters of the skull at this level on axial MRI (< $40 \%$ is considere normal, $40 \%-50 \%$ is considered borderline, and $>50 \%$ is hydrocephalus). ${ }^{26,45}$ These clinical and radiological parameters were collected at the time of diagnosis and subsequent follow-up (median number of follow-ups 2, range 1-4). Response to treatment was evaluated using criteria similar to those used in the RECIST (Response Evaluation Criteria in Solid Tumors) guidelines for solid tumors (version 1.1): ${ }^{15}$ response was defined as a decrease in tumor volume $\geq 30 \%$, progression if tumor volume increased by $\geq 20 \%$, and stable otherwise. As is the convention in brain radiosurgery studies, local control is traditionally reported as metastases that are stable or smaller after GKRS.

The GKRS technique at our center has been previously described. ${ }^{4}$ In brief, all patients had a preliminary preprocedural evaluation by a neurosurgeon and radiation oncologist to determine appropriateness for GKRS. On the day of treatment, volumetric MRI of the head was performed, followed by stereotactic CT scanning (Leksell Frame G). Target delineation was planned on the GammaPlan planning system (Elekta). Typically, the prescribed doses depended on the maximal dimension of the lesion according to standard practice at our institution and were consistent with the RTOG (Radiation Therapy Oncology Group) 90- 
Muhsen et al.

TABLE 1. Baseline patient demographics, disease characteristics, and radiosurgical parameters used in the treatment of LPFM

\begin{tabular}{|c|c|}
\hline & Value \\
\hline \multicolumn{2}{|l|}{ Age, yrs } \\
\hline Median & 61.5 \\
\hline Range & $29-94$ \\
\hline \multicolumn{2}{|l|}{ Sex, n (\%) } \\
\hline Female & $21(42.9)$ \\
\hline Male & $28(57.1)$ \\
\hline \multicolumn{2}{|l|}{ KPS score } \\
\hline Median & 90 \\
\hline Range & $60-90$ \\
\hline \multicolumn{2}{|l|}{ Follow-up period, mos } \\
\hline Median & 7.3 \\
\hline Range & $1.6-57.2$ \\
\hline \multicolumn{2}{|l|}{ Cerebellar metastases, $n$} \\
\hline Median & 1 \\
\hline Range & $1-4$ \\
\hline \multicolumn{2}{|l|}{ Overall no. of brain metastases } \\
\hline Median & 2 \\
\hline Range & $1-17$ \\
\hline \multicolumn{2}{|l|}{ Lesion diameter, $\mathrm{cm}$} \\
\hline Median & 2.6 \\
\hline Range & $2-5.07$ \\
\hline \multicolumn{2}{|l|}{ Primary tumor, $\mathrm{n}(\%)$} \\
\hline Lung & $22(44.9)$ \\
\hline Breast & $13(26.5)$ \\
\hline Colon & $5(10.2)$ \\
\hline $\mathrm{RCC}$ & $4(8.2)$ \\
\hline Melanoma & $2(4.1)$ \\
\hline Other & $3(6.1)$ \\
\hline \multicolumn{2}{|l|}{ LPFM location, $n(\%)$} \\
\hline Rt cerebellar hemisphere & $22(43.1)$ \\
\hline Lt cerebellar hemisphere & $21(41.2)$ \\
\hline Vermis & $8(15.7)$ \\
\hline \multicolumn{2}{|l|}{ Prescription dose to LPFM, Gy } \\
\hline Median & 18 \\
\hline Range & $12-24$ \\
\hline \multicolumn{2}{|l|}{ Conformality index for LPFM } \\
\hline Median & 1.497 \\
\hline Range & $1.115-2.495$ \\
\hline \multicolumn{2}{|l|}{ Isodose line for LPFM, \% } \\
\hline Median & 53 \\
\hline Range & $53-73$ \\
\hline
\end{tabular}

05 dosing schedule and guidelines. ${ }^{34,35}$ Of note, 1 lesion that measured $5.07 \mathrm{~cm}$ in diameter was treated with $12 \mathrm{~Gy}$. In our series the median isodose line used was 53\% (range $50 \%-64 \%$ ), and the median conformality index was 1.497 (range 1.115-2.495). All lesions were treated with a single fraction. Prior to treatment, all plans were reviewed and
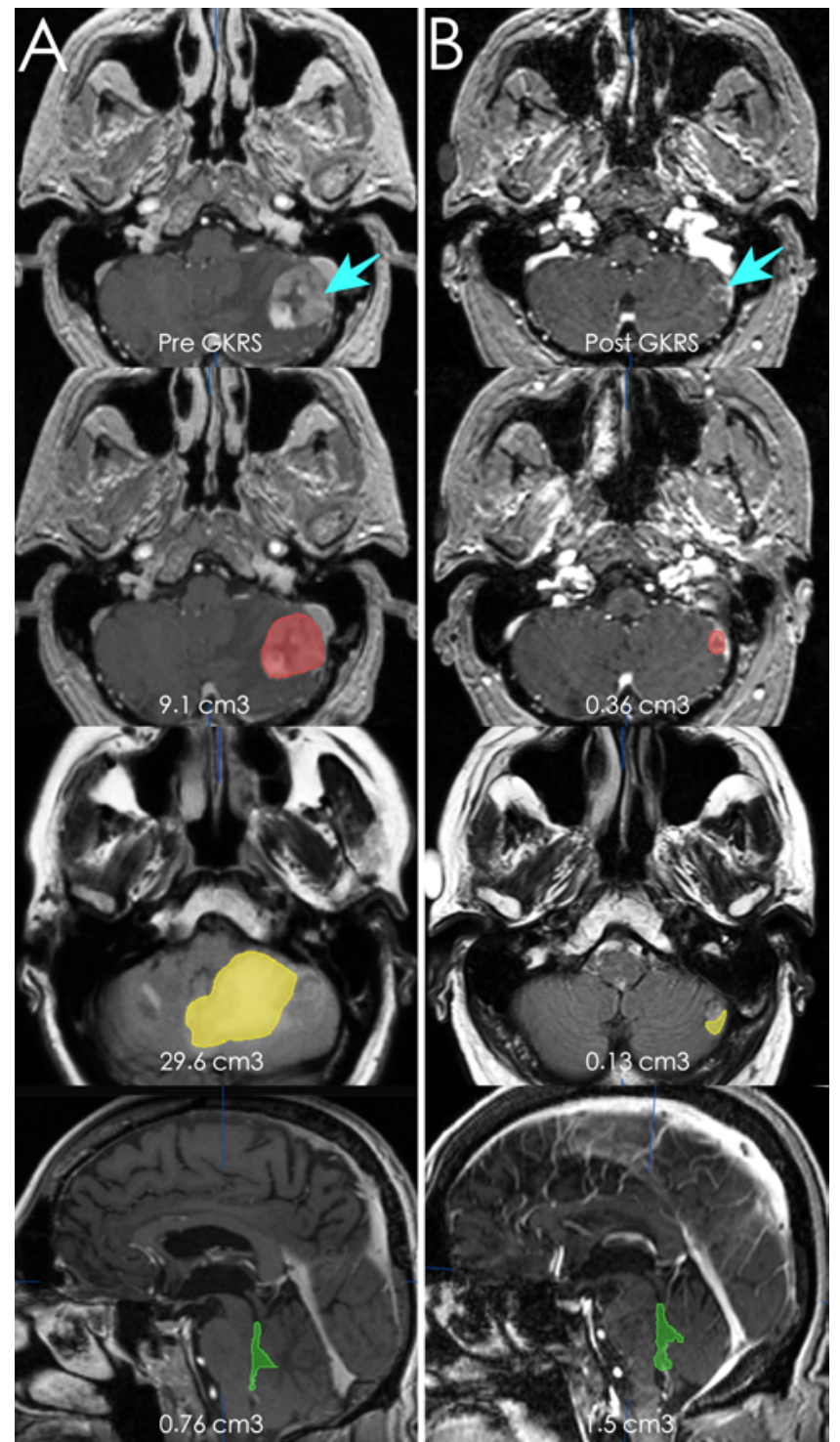

FIG. 1. Contours demonstrating volumes of tumor (red), PTE (yellow), and fourth ventricle (green) on MR images obtained pre-GKRS $(\mathbf{A})$ and post-GKRS (B; at the first follow-up). Blue arrows point to the tumor location.

approved by a neurosurgeon, radiation oncologist, and medical physicist. Most patients had follow-up scans in the 6- to 12-week window, and the adverse radiation effects were graded using CTCAE (Common Terminology Criteria for Adverse Events) v5.0 (https://ctep.cancer.gov/ protocolDevelopment/electronic_applications/ctc.htm).

Statistical analysis was performed using the Wilcoxon signed-rank test and t-test. Overall survival (OS) was analyzed using the Kaplan-Meier method. All statistical analyses were performed using $\mathrm{R}$ version 3.5.0. Statistical significance was set at $\mathrm{p}<0.05$.

\section{Results}

Complete patient demographics, disease characteristics, and treatment details were obtained from 49 patients 
TABLE 2. Clinical presentation of patients at the time of GKRS treatment

\begin{tabular}{cc}
\hline & No. of Patients (\%) \\
\hline Asymptomatic & $18(37)$ \\
\hline Nonspecific symptoms & $19(39)$ \\
\hline Symptomatic & $12(24)$ \\
\hline Gait imbalance & $8(67)$ \\
\hline Headache & $6(50)$ \\
\hline Nausea/vomiting & $2(17)$ \\
\hline Dysarthria & $1(8)$ \\
\hline Dysmetria & $6(50)$ \\
\hline Dysdiadochokinesia & $6(50)$ \\
\hline
\end{tabular}

who had 51 lesions and met the inclusion and exclusion criteria, which are summarized in Table 1 . The median age was 61.5 years (range 29-94 years). Twenty-eight patients $(57.1 \%)$ were male. The median KPS score at the time of GKRS was 90 (range 60-90), the median number of cerebellar metastases was 1 (range 1-4), and the median number of total brain metastases for each patient was 2 (range 1-17). The median prescription dose to the lesion was $18 \mathrm{~Gy}$, and the median lesion diameter was $2.6 \mathrm{~cm}$ (range $2.0-5.07 \mathrm{~cm}$ ). The most common primary source was the lung in 22 patients $(44.9 \%)$. Breast carcinoma was the second most common pathology treated, with 13 patients $(26.5 \%)$; 5 patients $(10.2 \%)$ had colon cancer, 4 patients $(8.2 \%)$ had renal cell carcinoma (RCC), 2 patients $(4.1 \%)$ had melanoma, and 1 patient each $(2 \%)$ had a neuroendocrine tumor, esophageal cancer, and laryngeal cancer. Twenty patients $(40.8 \%)$ had a history of prior WBRT, including 5 patients (10.2\%) with small cell lung carcinoma.

The location of LPFMs was the right cerebellar hemisphere in 22 cases, left cerebellar hemisphere in 21 cases, and vermis in 8 cases. Seventeen lesions $(33.3 \%)$ were single LPFMs, and 32 lesions (62.7\%) were LPFMs with other posterior fossa or supratentorial metastases (STMs). All non-LPFMs and STMs were treated with GKRS according to the RTOG $90-05$ dosing schedule, ${ }^{35}$ which is standard at our institution. Two patients had 2 LPFMs: one had 2 LPFMs in the left cerebellar hemisphere and the other had bilateral LPFMs. In addition to cerebellar disease, 31 patients $(63.3 \%)$ had STMs, of whom $10(32.3 \%)$ had a single STM, 11 patients $(35.5 \%)$ had 2 STMs, 5 patients $(16.1 \%)$ had 3 STMs, 2 patients (12.9\%) had 4 STMs, and 1 patient (3.2\%) had 14 STMs. Eighteen patients $(36.73 \%)$ were asymptomatic at the time of GKRS, 19 patients (38.77\%) had nonspecific signs or symptoms, and 12 patients $(24.48 \%)$ had signs and symptoms of cerebellar disease (24.5\%). The clinical presentation of these patients is summarized in Table 2.

As this initially was a novel approach at our institution, we were more cautious and as a standard practice we admitted patients for 48 hours to monitor signs and symptoms of hydrocephalus. None of the patients required any temporary or permanent surgical intervention or CSF diversion procedure during that observation period. The median tumor, PTE, and fourth ventricle volumes at diagnosis were $4.96 \mathrm{~cm}^{3}$ (range 1.4-21.1 $\mathrm{cm}^{3}$ ), $14.98 \mathrm{~cm}^{3}$ (range $0.6-71.8 \mathrm{~cm}^{3}$ ), and $1.23 \mathrm{~cm}^{3}\left(\right.$ range $0.3-3.2 \mathrm{~cm}^{3}$ ), respectively. For statistical analysis, in patients with multiple LPFMs, the total tumor burden and PTE burden were calculated by combining the posterior fossa tumors and their accompanying PTE for each patient. The median volume of tumor plus PTE at diagnosis was $19.61 \mathrm{~cm}^{3}$ (range 2.88-91.28 $\mathrm{cm}^{3}$ ).

At the first follow-up (median 8.1 weeks, range 6-12 weeks), combined tumor and PTE volumes decreased in 47 lesions $(92.15 \%)$ and increased in $4(7.84 \%)$. The median tumor volume was $2.05 \mathrm{~cm}^{3}$ (range $0.20-15.79 \mathrm{~cm}^{3}$ ), the median PTE was $3.27 \mathrm{~cm}^{3}$ (range $0.01-46.34 \mathrm{~cm}^{3}$ ), and the median fourth ventricle volume was $1.77 \mathrm{~cm}^{3}$ (range $\left.0.49-4.09 \mathrm{~cm}^{3}\right)$. The median tumor volume decreased by $58.66 \%$ (range $-96.95 \%$ to $+48.69 \%, \mathrm{p}<0.001$ ), median PTE decreased by $78.10 \%$ (range $-99.92 \%$ to $+198.35 \%$, $\mathrm{p}<0.001$ ), and the fourth ventricle increased by $24.97 \%$ (range $-37.96 \%$ to $+545.6 \%, p<0.001$ ) (Fig. 2). The median follow-up duration was 7.3 months (range 1.6-57.2 months). The local control rate at first follow-up was 98.1\% (76.5\% decreased, $21.6 \%$ remained stable, and $1.9 \%$ progressed). None of the patients in our series developed radiological evidence of leptomeningeal disease in the follow-up period. The median KPS score at diagnosis of the study population was 90 (range 60-90). A KPS score of 90 was seen in $27(55.1 \%)$ of 49 patients; 12 (24.49\%), 9 $(18.37 \%)$, and 1 (2.04\%) patients had KPS scores of 80,70, and 60, respectively. At the first follow-up, the overall KPS score improved in 6 patients $(12.24 \%)$, was unchanged in $36(73.46 \%)$, and decreased in $7(14.28 \%)$. Among the 6 patients with improvement in KPS scores, the scores in 5 had increased by 10 points and in 1 patient by 20 points. Among the 7 patients with a decrease in KPS score, 6 patients' KPS scores decreased by 10 and in the other patient by 40 (which was due to progression of systemic disease). Overall, there was no statistically significant change in KPS score $(\mathrm{p}=0.80)$ at follow-up. The median OS was 8.36 months, which was plotted on a Kaplan-Meier curve (Fig. 3). At the final follow-up (median 7.3 months), we observed a significant decrease in the median tumor (71.33\%, range $-99.66 \%$ to $+128.38 \%)$ and PTE $(79.96 \%$, range $-99.97 \%$ to $+199.04 \%$ ) volumes and an increase in the fourth ventricle volume $(38.20 \%$, range $-41.14 \%$ to $+655.08 \%$ ) (Fig. 4).

Clinical symptoms were noted in detail before and after GKRS and at every subsequent follow-up. Patients were grouped into those with cerebellar symptoms, those who were asymptomatic, and those with nonspecific neurological symptoms such as generalized weakness, occasional headaches, and treatment-related neuropathy. At the first follow-up (6-12 weeks), clinical improvement was seen in 6 of the 12 patients who presented with cerebellar symptoms, 5 patients remained unchanged, and 1 patient worsened (developed new-onset nonspecific symptoms thought to be secondary to progression of their systemic disease). One of the 19 patients in the nonspecific-symptoms group developed new-onset cerebellar symptoms at the first follow-up. Overall, 3 (6\%) of 49 patients had worsening of their neurological symptoms at first follow-up. None of the 


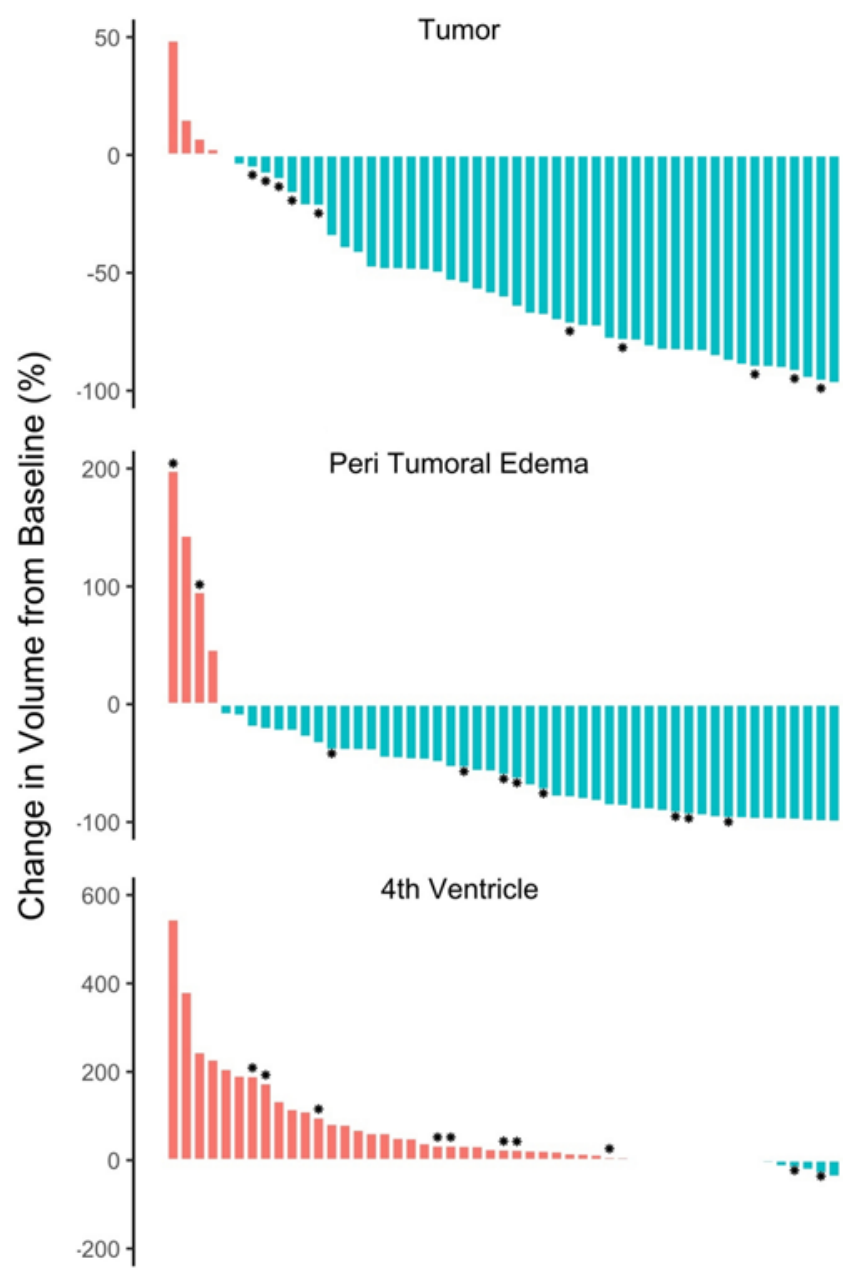

FIG. 2. Waterfall plots demonstrating the change in tumor, PTE, and fourth ventricle volumes at the first follow-up. The median tumor volume decreased by $58.66 \%$ (range $-96.95 \%$ to $+48.69 \%, p<0.001$ ), median PTE decreased by $78.10 \%$ (range $-99.92 \%$ to $+198.35 \%, p<0.001$ ), and fourth ventricle increased by $24.97 \%$ (range $-37.96 \%$ to $+545.6 \%$, $p<0.001$ ). *Radioresistant tumors: triple-negative breast cancer, RCC, melanoma, and colon cancer. There was no statistically significant difference in the response rates of radioresistant tumors versus radiosensitive tumors at the first follow-up ( $p=0.058$ ) or last follow-up ( $p=0.11)$.

patients in our series had evidence of hydrocephalus based on clinical and radiological evaluation (as evidenced by the normal frontal horn to internal diameter ratio [frontal horn/inner diameter ratio]), at the time of diagnosis and at first follow-up ( $\mathrm{p}=0.11)$ (Fig. 5). After GKRS, a tapering dose of steroids was given to 47 patients, of whom 14 $(29.79 \%)$ required steroids beyond our typical steroid taper regimen (tapered over a maximum period of 4 weeks). There was no statistically significant correlation between change in tumor and PTE volume at diagnosis and at the first follow-up with more prolonged use of steroids versus a routine taper dose of steroids $(p=0.40)$ Steroid-related complications were seen in 5 patients; 3 of them had worsening of preexisting type II diabetes mellitus and 2 developed steroid myopathy and cushingoid features with weight gain and facial puffiness. None of the patients required bevacizumab (anti-vascular endothelial growth

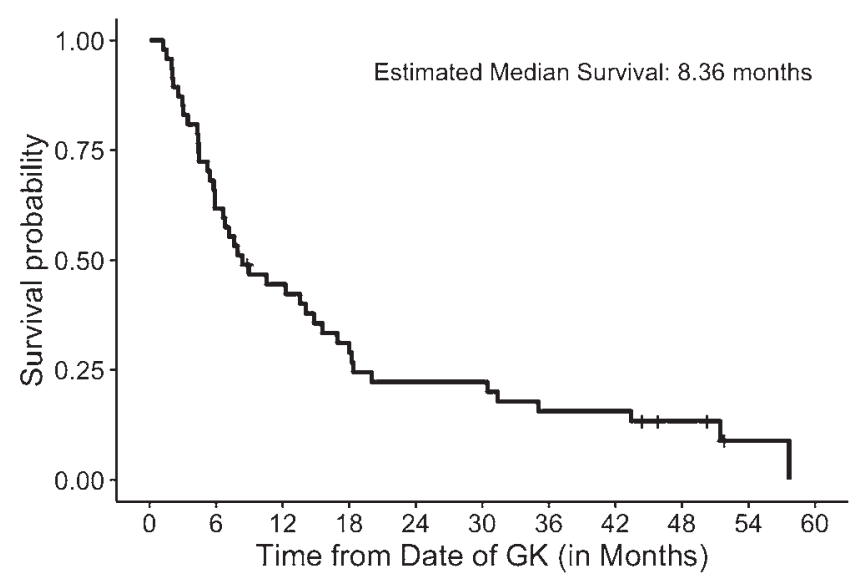

FIG. 3. Kaplan-Meier curve for overall survival in patients with LPFMs undergoing GKRS.

factor) in the post-GKRS period for treatment of tumorrelated edema; however, 1 patient required bevacizumab as an adjuvant in the treatment of his primary tumor.

In the event of increasing lesion size at first follow-up after GKRS, radiological and/or pathological information was used to discriminate progression and radiation necrosis. Specifically, stabilization or shrinkage of a previously enlarging lesion and/or decreased cerebral blood volume on perfusion MRI was considered related to radiation necrosis, whereas continuous increase in mass and/ or increased cerebral blood volume was considered progression. Three patients (6.1\%) developed symptomatic radiation necrosis. One of these 3 patients underwent laser interstitial thermal therapy 14 months post-GKRS, the second patient was treated with bevacizumab and required a ventriculoperitoneal shunt 23 months after GKRS, and the third patient had symptomatic radiation necrosis 15 months after GKRS and was treated with steroids alone. Two other patients had asymptomatic radiation necrosis and required no treatment for it. Two patients developed new cerebellar metastasis during the follow-up period and were treated with GKRS.

\section{Discussion}

Brain metastases are considered an advanced stage of systemic malignancy, and the treatment of large brain metastases remains challenging due to many potential complications, especially if the lesions are located in the posterior fossa. ${ }^{1,6,7,12}$ There are many treatment options available today for posterior fossa metastases, including surgery, WBRT, and GKRS, either alone or in combination. ${ }^{2,3,6,8,16}$ However, an appropriate treatment option for a patient with brain metastasis should be decided after considering various factors, such as the underlying primary pathology, the location of the lesion, the size of the lesion, the clinical status of the patient, and the burden of the systemic disease.

In patients with brain metastases, the major advantage of GKRS over surgery is that it is less invasive and not associated with significant recovery time so that systemic therapy can be administered sooner than would be oth- 

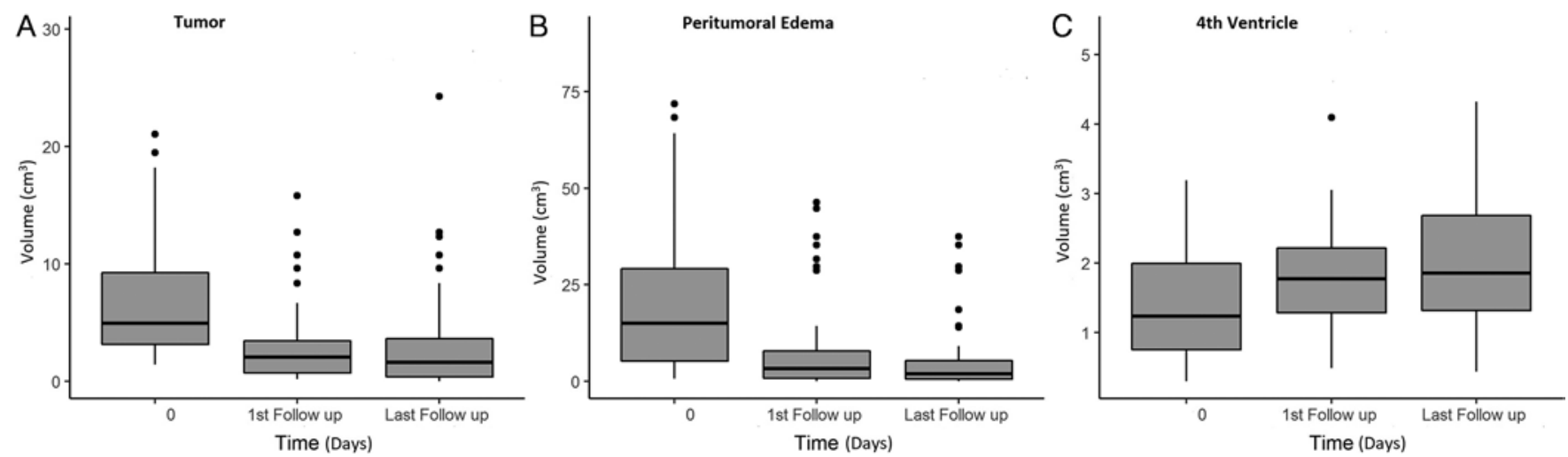

FIG. 4. Box plots demonstrating the change in tumor (A), PTE (B), and fourth ventricle (C) volumes at the first (median 8.1 weeks) and final (median 7.3 months) follow-up.

erwise possible following open surgical intervention. In a retrospective case series of 92 patients with posterior fossa metastases managed surgically, patients with posterior fossa metastatic lesions had a poor prognosis with a median OS of 6 months, and surgical management was not found to be a significant prognostic factor. ${ }^{44}$ In addition, GKRS has the potential to treat multiple lesions in the same session and to treat deep-seated brain lesions. 7,8 , 20,28,33 Furthermore, GKRS was also found to be associated with a lower incidence of leptomeningeal disease when compared with surgery, whereby GKRS obviated the potentially serious complications associated with surgery and resulted in an equivalent OS rate and improved initial performance status. ${ }^{40}$

WBRT is another modality used to treat patients with brain metastases, especially those with multiple lesions and those who are poor surgical candidates. Currently,

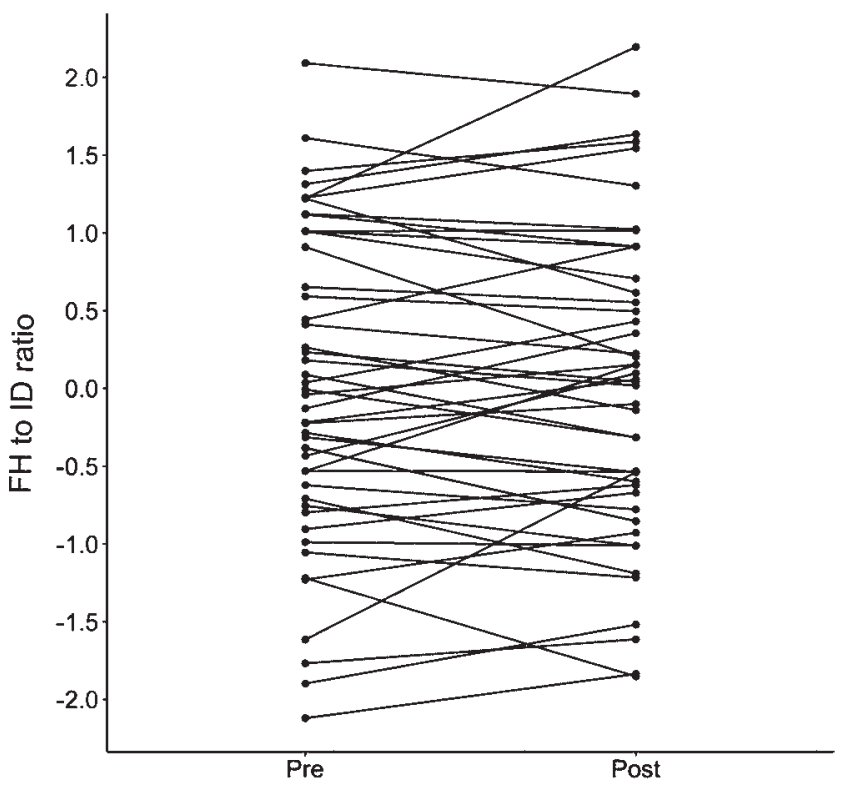

FIG. 5. Changes in the pre- and post-GKRS ratio of the largest width of the frontal horn (FH) to the internal diameter (ID) from inner table to inner table at this level, showing that there was no statistically significant $(p=0.11)$ increase in the size of the lateral horns at the first follow-up. in many centers, radiosurgery has overtaken WBRT as a preferred modality to treat metastatic lesions, due to concerns for increased cognitive toxicity in patients undergo-

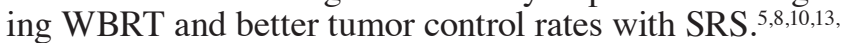
${ }^{36,46}$ In a randomized clinical trial investigating the effect of SRS alone in comparison to SRS with WBRT on cognitive function in patients with brain metastases, the use of SRS alone resulted in less cognitive deterioration without a significant difference in OS rate. ${ }^{8}$ Such findings suggest that SRS alone can be a preferred therapeutic option for patients with brain metastases amenable to radiosurgery. Moreover, in contrast to SRS, WBRT was positively correlated with worsened PTE at follow-up, and hence these patients required a prolonged duration of steroid therapy. ${ }^{18,27}$ These studies also demonstrated that, in contrast to WBRT, SRS was efficacious in controlling PTE ${ }^{18,27}$

In comparison to other treatment modalities such as resection, SRS demonstrates an equivalent local tumor control in most patients and a decreased need for the interruption of chemotherapy for the systemic disease control. 5,8,29,37 Tumor control is an important outcome measure in patients with brain metastases; however, control of the associated mass effect is important for treatment safety and improves the neurological deficits secondary to the tumor and associated PTE, and hence is important for improving the patient's quality of life. ${ }^{27,37}$ With the paucity of available literature on the effect of SRS on PTE around LPFMs, our aim was to study the impact of this in patients treated at our institution. In areas in which the tumors were not restricted to the posterior fossa, improvement in neurological function has been observed to be associated with a reduction in PTE after GKRS..$^{27,37,38}$ In the series by Mori et al., improvement in motor weakness was seen in 5 of 20 patients after SRS. ${ }^{23}$ Of the 5 patients in their series who improved, 3 of them had improvement in PTE on MRI at the first follow-up. ${ }^{23}$ Moreover, longer survival has also been observed in patients who had better control of PTE at follow-up. ${ }^{18,27,37,38}$ Therefore, effective management of PTE is correlated with improved overall patient outcomes following GKRS or SRS therapy.

The management of PTE may play an important role in preventing surgical intervention to address compression of the fourth ventricle leading to hydrocephalus. ${ }^{30}$ Suboccipital craniectomy with dural opening for decompression 
followed by shunting for CSF diversion is associated with significant risks of shunt infections and/or malfunctions requiring revisions and leads to a prolonged recovery period wherein chemotherapy must be delayed or deferred. ${ }^{30,31}$ In addition, rare complications, such as extracranial, peritoneal seeding of tumor cells through ventriculoperitoneal shunts leading to the formation of malignant CSF ascites, and intratumoral hemorrhage immediately following a shunt procedure, have been reported. ${ }^{19,21}$ Such potential complications can significantly exacerbate patient morbidity, performance status, and overall clinical outcomes and are avoided by effective PTE control and management of fourth ventricle compression in GKRS.

Another factor that influences the efficacy and safety of GKRS seen in previous studies is histology of the primary tumor. ${ }^{37,38}$ Shuto et al. studied the effect of GKRS on control of cerebral metastases and the PTE around them in 280 patients with various-sized metastases and primary pathologies that were located in both the supratentorial and infratentorial compartments. ${ }^{37}$ Their study demonstrated that PTE associated with metastases from primary lung and breast malignancies (non-RCC) was less frequently found at initial diagnosis and was better controlled after GKRS on follow-up MRI than PTE associated with RCC metastases.$^{37}$ In a subsequent study, the effect of resection alone was compared with GKRS in patients with large RCC metastases that measured $>2 \mathrm{~cm}$ in maximal diameter and were associated with extensive PTE. ${ }^{38}$ The PTE improved in all of their patients who underwent resection, whereas only $63.8 \%$ of patients who underwent GKRS had control of PTE. ${ }^{38}$ Based on these observations, it was concluded that RCC as the primary histology is a poor prognostic factor for PTE control. ${ }^{37,38}$ Given that there were only 4 patients with RCC metastases in our series, and 1 of them had worsening of PTE and the other 3 had reduction of PTE at the first follow-up after GKRS, we cannot draw definitive conclusions regarding the efficacy and safety of GKRS in RCC metastatic patients in terms of local tumor control and PTE management; this will require further larger series.

High-dose steroids are widely utilized after GKRS as a medical therapy to control PTE in metastatic lesions. In the available literature, conflicting results are found with respect to the use of steroids in the control of PTE. Pan et al. observed control of PTE in patients who received steroids after GKRS, whereas Shuto et al. demonstrated that steroid administration was not correlated with PTE control on follow-up MRI. ${ }^{27,37,38}$ Similarly, in our study, we did not find a significant correlation between the use of steroids after GKRS and control of PTE. Previous studies have shown that significant preexisting edema did not influence the tumor response or clinical outcome, and resolution of edema was related to better quality of life but not to longer survival. ${ }^{27}$ In our series of LPFMs with preexisting surrounding PTE, it was found that the size of the lesion does not always correlate directly with the severity of clinical symptoms; hence, a relatively conservative approach, like GKRS, performed under close observation could be justified.

While the results of this study are encouraging, our analysis was limited by the retrospective study design, small sample size, and various tumor pathologies, including a variety of relatively radiosensitive and radioresistant tumors. Also, we did not test for differences between the patients who did not meet the inclusion criteria and those who were not included due to the absence of repeat MRI and/or follow-up, potentially introducing a selection bias. We recognize that while the clinically meaningful data were delineated and reviewed, long-term potential differences in clinical outcomes were not reviewed, given the variability of the long-term follow-up in this cohort. Additional prospective studies with a larger sample size investigating the correlation with clinical outcome changes are warranted to further analyze the role of GKRS in the management of large posterior fossa metastases and the associated edema. Finally, we cannot emphasize enough that this is a highly selected group of patients; strict criteria were maintained during case selection and these cases were performed in a setting with vast experience in GKRS. The majority of patients in this group were only minimally symptomatic and patients with symptomatic hydrocephalus, hemorrhage, and presence of symptoms related to brainstem compression were promptly excluded. Moreover, until larger prospective studies substantiate the safety and efficacy of GKRS in LPFM, caution and judicious patient selection should be exercised in selecting this approach.

\section{Conclusions}

In this retrospective analysis of patients with LPFMs, GKRS resulted in a statistically significant reduction in PTE and tumor size as well as the desired increase in the size of the fourth ventricle (all $p<0.001$ ) at both the first posttreatment follow-up and final follow-up without significant treatment-related toxicity. Hence, GKRS monotherapy can be considered a potential treatment option for patients with LPFMs, including those lesions that are associated with marked PTE and compression/distortion of the fourth ventricle.

\section{References}

1. Al-Shamy G, Sawaya R: Management of brain metastases: the indispensable role of surgery. J Neurooncol 92:275-282, 2009

2. Alexander E III, Loeffler JS: The case for radiosurgery. Clin Neurosurg 45:32-40, 1999

3. Andrews DW, Scott CB, Sperduto PW, Flanders AE, Gaspar LE, Schell MC, et al: Whole brain radiation therapy with or without stereotactic radiosurgery boost for patients with one to three brain metastases: phase III results of the RTOG 9508 randomised trial. Lancet 363:1665-1672, 2004

4. Angelov L, Mohammadi AM, Bennett EE, Abbassy M, Elson P, Chao ST, et al: Impact of 2-staged stereotactic radiosurgery for treatment of brain metastases $\geq 2 \mathrm{~cm}$. J Neurosurg 129:366-382, 2018

5. Aoyama H, Tago M, Shirato H: Stereotactic radiosurgery with or without whole-brain radiotherapy for brain metastases: secondary analysis of the JROSG 99-1 randomized clinical trial. JAMA Oncol 1:457-464, 2015

6. Bennani O, Derrey S, Langlois O, Castel H, Laquerriere A, Freger $\mathrm{P}$, et al: Brain metastasis from renal cell carcinoma. Neurochirurgie 60:12-16, 2014

7. Bhangoo SS, Linskey ME, Kalkanis SN: Evidence-based 
guidelines for the management of brain metastases. Neurosurg Clin N Am 22:97-104, viii, 2011

8. Brown PD, Jaeckle K, Ballman KV, Farace E, Cerhan JH, Anderson SK, et al: Effect of radiosurgery alone vs radiosurgery with whole brain radiation therapy on cognitive function in patients with 1 to 3 brain metastases: a randomized clinical trial. JAMA 316:401-409, 2016

9. Buatti JM, Friedman WA, Bova FJ, Mendenhall WM: Treatment selection factors for stereotactic radiosurgery of intracranial metastases. Int J Radiat Oncol Biol Phys 32:11611166,1995

10. Chang EL, Wefel JS, Hess KR, Allen PK, Lang FF, Kornguth DG, et al: Neurocognition in patients with brain metastases treated with radiosurgery or radiosurgery plus whole-brain irradiation: a randomised controlled trial. Lancet Oncol 10:1037-1044, 2009

11. Chidel MA, Suh JH, Reddy CA, Chao ST, Lundbeck MF, Barnett GH: Application of recursive partitioning analysis and evaluation of the use of whole brain radiation among patients treated with stereotactic radiosurgery for newly diagnosed brain metastases. Int J Radiat Oncol Biol Phys 47:993-999, 2000

12. Cho KH, Hall WA, Gerbi BJ, Higgins PD, Bohen M, Clark HB: Patient selection criteria for the treatment of brain metastases with stereotactic radiosurgery. J Neurooncol 40:7386, 1998

13. Crossen JR, Garwood D, Glatstein E, Neuwelt EA: Neurobehavioral sequelae of cranial irradiation in adults: a review of radiation-induced encephalopathy. J Clin Oncol 12:627-642, 1994

14. Dasararaju R, Mehta A: Current advances in understanding and managing secondary brain metastasis. CNS Oncol 2:75-85, 2013

15. Eisenhauer EA, Therasse P, Bogaerts J, Schwartz LH, Sargent $\mathrm{D}$, Ford R, et al: New response evaluation criteria in solid tumours: revised RECIST guideline (version 1.1). Eur J Cancer 45:228-247, 2009

16. Fuentes R, Bonfill X, Exposito J: Surgery versus radiosurgery for patients with a solitary brain metastasis from non-small cell lung cancer. Cochrane Database Syst Rev (1):CD004840, 2006

17. Gondi V, Mehta MP: Novel insights into the management of brain metastases. Curr Opin Neurol 23:556-562, 2010

18. Hawighorst H, Essig M, Debus J, Knopp MV, EngenhartCabilic R, Schönberg SO, et al: Serial MR imaging of intracranial metastases after radiosurgery. Magn Reson Imaging 15:1121-1132, 1997

19. Kasliwal MK, Agrawal D, Sharma BS: Fatal intratumoral hemorrhage following ventriculo-peritoneal shunt. Turk Neurosurg 18:436-438, 2008

20. Kondziolka D, Niranjan A, Flickinger JC, Lunsford LD: Radiosurgery with or without whole-brain radiotherapy for brain metastases: the patients' perspective regarding complications. Am J Clin Oncol 28:173-179, 2005

21. Low YY, Thomas J, Wan WK, Ng WH: Brain metastases as a cause of malignant cerebrospinal fluid ascites: case report and review of the literature. CNS Oncol 1:29-37, 2012

22. Metellus P, Bialecki E, Le Rhun E, Dhermain F: Neurosurgi$\mathrm{cal}$ and radiosurgical decision making in brain metastasis patients in the area of targeted therapies? Linchuang Zhongliuxue Zazhi 4:19, 2015

23. Mori Y, Kondziolka D, Flickinger JC, Logan T, Lunsford LD: Stereotactic radiosurgery for brain metastasis from renal cell carcinoma. Cancer 83:344-353, 1998

24. Naidich TP, Duvernoy HM, Delman BN, Sorensen AG, Kollias SS, Haacke EM: Duvernoy's Atlas of the Human Brain Stem and Cerebellum: High-Field MRI, Surface Anatomy, Internal Structure, Vascularization and 3 D Sectional Anatomy. Berlin: Springer Science \& Business Media, 2009
25. Niranjan A, Lunsford LD, Emerick RL: Stereotactic radiosurgery for patients with metastatic brain tumors: development of a consensus radiosurgery guideline recommendation. Prog Neurol Surg 25:123-138, 2012

26. Pakkenberg B, Boesen J, Albeck M, Gjerris F: Unbiased and efficient estimation of total ventricular volume of the brain obtained from CT-scans by a stereological method. Neuroradiology 31:413-417, 1989

27. Pan HC, Sun MH, Chen CC, Chen CJ, Lee CH, Sheehan J: Neuroimaging and quality-of-life outcomes in patients with brain metastasis and peritumoral edema who undergo Gamma Knife surgery. J Neurosurg 109 Suppl:90-98, 2008

28. Patel TR, McHugh BJ, Bi WL, Minja FJ, Knisely JP, Chiang VL: A comprehensive review of MR imaging changes following radiosurgery to 500 brain metastases. AJNR Am J Neuroradiol 32:1885-1892, 2011

29. Patil CG, Pricola K, Garg SK, Bryant A, Black KL: Whole brain radiation therapy (WBRT) alone versus WBRT and radiosurgery for the treatment of brain metastases. Cochrane Database Syst Rev (6):CD006121, 2010

30. Reddy GK, Bollam P, Caldito G, Willis B, Guthikonda B, Nanda A: Ventriculoperitoneal shunt complications in hydrocephalus patients with intracranial tumors: an analysis of relevant risk factors. J Neurooncol 103:333-342, 2011

31. Rinaldo L, Brown D, Lanzino G, Parney IF: Outcomes following cerebrospinal fluid shunting in high-grade glioma patients. J Neurosurg 129:984-996, 2018

32. Ryan R, Booth S, Price S: Corticosteroid-use in primary and secondary brain tumour patients: a review. J Neurooncol 106:449-459, 2012

33. Sanghavi SN, Miranpuri SS, Chappell R, Buatti JM, Sneed PK, Suh JH, et al: Radiosurgery for patients with brain metastases: a multi-institutional analysis, stratified by the RTOG recursive partitioning analysis method. Int J Radiat Oncol Biol Phys 51:426-434, 2001

34. Shaw E, Scott C, Souhami L, Dinapoli R, Bahary JP, Kline $\mathrm{R}$, et al: Radiosurgery for the treatment of previously irradiated recurrent primary brain tumors and brain metastases: initial report of radiation therapy oncology group protocol (90-05). Int J Radiat Oncol Biol Phys 34:647-654, 1996

35. Shaw E, Scott C, Souhami L, Dinapoli R, Kline R, Loeffler J, et al: Single dose radiosurgical treatment of recurrent previously irradiated primary brain tumors and brain metastases: final report of RTOG protocol 90-05. Int J Radiat Oncol Biol Phys 47:291-298, 2000

36. Sheline GE, Wara WM, Smith V: Therapeutic irradiation and brain injury. Int J Radiat Oncol Biol Phys 6:1215-1228, 1980

37. Shuto T, Matsunaga S, Inomori S, Fujino H: Efficacy of gamma knife surgery for control of peritumoral oedema associated with metastatic brain tumours. J Neurol Neurosurg Psychiatry 79:1061-1065, 2008

38. Shuto T, Matsunaga S, Suenaga J, Inomori S, Fujino H: Treatment strategy for metastatic brain tumors from renal cell carcinoma: selection of gamma knife surgery or craniotomy for control of growth and peritumoral edema. J Neurooncol 98:169-175, 2010

39. Siegfried J, Wellis G, Scheib S, Haller D, Landolt AM, Lomax NJ, et al: [Palliative treatment of brain metastases with Gamma Knife.] Ther Umsch 58:413-418, 2001 (German)

40. Siomin VE, Vogelbaum MA, Kanner AA, Lee SY, Suh JH, Barnett GH: Posterior fossa metastases: risk of leptomeningeal disease when treated with stereotactic radiosurgery compared to surgery. J Neurooncol 67:115-121, 2004

41. Sneed PK, Suh JH, Goetsch SJ, Sanghavi SN, Chappell R, Buatti JM, et al: A multi-institutional review of radiosurgery alone vs. radiosurgery with whole brain radiotherapy as the initial management of brain metastases. Int J Radiat Oncol Biol Phys 53:519-526, 2002 
42. Soffietti R, Rudā R, Mutani R: Management of brain metastases. J Neurol 249:1357-1369, 2002

43. Sperduto PW, Berkey B, Gaspar LE, Mehta M, Curran W: A new prognostic index and comparison to three other indices for patients with brain metastases: an analysis of 1,960 patients in the RTOG database. Int J Radiat Oncol Biol Phys 70:510-514, 2008

44. Sunderland GJ, Jenkinson MD, Zakaria R: Surgical management of posterior fossa metastases. J Neurooncol 130:535542,2016

45. Synek V, Reuben JR: The ventricular-brain ratio using planimetric measurement of EMI scans. Br J Radiol 49:233-237, 1976

46. Tallet AV, Azria D, Barlesi F, Spano JP, Carpentier AF, Gonçalves A, et al: Neurocognitive function impairment after whole brain radiotherapy for brain metastases: actual assessment. Radiat Oncol 7:77, 2012

\section{Disclosures}

Dr. Mohammadi: consultant for Monteris Medical. Dr. Suh: consultant for Chrysalis Biotherapeutics.

\section{Author Contributions}

Conception and design: Angelov, Muhsen, Joshi. Acquisition of data: Muhsen, Joshi, Lee, Thapa, Borghei-Razavi. Analysis and interpretation of data: Angelov, Muhsen, Joshi, Lee, Thapa. Drafting the article: Angelov, Muhsen, Joshi, Lee, Thapa. Critically revising the article: Angelov, Joshi, Borghei-Razavi, Jia, Barnett, Chao, Mohammadi, Suh. Reviewed submitted version of manuscript: Angelov, Joshi, Chao, Mohammadi, Suh, Vogelbaum. Approved the final version of the manuscript on behalf of all authors: Angelov. Statistical analysis: Muhsen, Joshi, Jia. Administrative/technical/material support: Angelov, Muhsen, Joshi, Barnett. Study supervision: Angelov, Muhsen, Joshi.

\section{Supplemental Information}

\section{Online-Only Content}

Supplemental material is available with the online version of the article.

Supplementary Table 1. https://thejns.org/doi/suppl/10.3171/ 2019.11.JNS191485.

\section{Current Affiliations}

Dr. Lee: Department of Neurosurgery, Barrow Neurological Institute, Phoenix, AZ.

\section{Correspondence}

Lilyana Angelov: The Rose Ella Burkhardt Brain Tumor \& Neuro-Oncology Center, Neurological and Taussig Cancer Institute, Cleveland Clinic, Cleveland, OH. angelol@ccf.org. 